\title{
Rougher is more slippery: How adhesive friction decreases with increasing surface roughness due to the suppression of capillary adhesion
}

\author{
Feng-Chun Hsia $\odot,{ }^{1,2}$ Steve Franklin $\odot,{ }^{1,3}$ Pierre Audebert $\odot,{ }^{4}$ Albert M. Brouwer, ${ }^{1,5}$ Daniel Bonn, ${ }^{2}$ and Bart Weber ${ }^{1,2, *}$ \\ ${ }^{1}$ Advanced Research Center for Nanolithography (ARCNL), Science Park 106, 1098 XG Amsterdam, Netherlands \\ ${ }^{2}$ Van der Waals-Zeeman Institute, Institute of Physics, University of Amsterdam, Science Park 904, 1098 XH Amsterdam, Netherlands \\ ${ }^{3}$ Department of Materials Science and Engineering and Mechanical Engineering, \\ The University of Sheffield, Sheffield S1 3JD, United Kingdom \\ ${ }^{4}$ Laboratoire de Photophysique et Photochemie Supramoléculaires et Macromoléculaires (PPSM), École normale supérieure (ENS) Cachan, \\ Centre national de la recherche scientifique (CNRS), Université Paris-Saclay, 94235 Cachan, France \\ ${ }^{5}$ Van 't Hoff Institute for Molecular Sciences, University of Amsterdam, Science Park 904, 1098 XH Amsterdam, Netherlands
}

(Received 3 August 2021; revised 28 September 2021; accepted 2 December 2021; published 21 December 2021)

\begin{abstract}
Friction originates at the area of real contact which depends on the (changing) surface topography. Observing and measuring the area of real contact at multi-asperity interfaces is difficult, making it challenging to quantitatively study the interplay between the frictional force and surface topography. In this paper, we systematically manipulate surface topography and use a fluorescence microscopy-based contact visualization technique to reveal this interplay. We demonstrate good agreement between elastoplastic boundary element method contact calculations and experimental visualization of the area of real contact. While the area of real contact and thus contact pressure could be varied by a factor of 4 through control of the surface topography, this had only a modest effect on the coefficient of friction $(\mathrm{CoF})$. We do find a small but systematic increase in the proportionality constant between frictional force and normal force $(\mathrm{CoF})$ with decreasing surface roughness. The observation that smoother surfaces have a greater $\mathrm{CoF}$ is due to capillary adhesion between the two surfaces. We quantitatively model this behavior using a simple capillary adhesion model without adjustable parameters. Our results provide quantitative insights into the interplay between contact mechanics, friction, and capillary adhesion. A predictive understanding of this interplay is essential to demanding applications such as precision positioning.
\end{abstract}

DOI: 10.1103/PhysRevResearch.3.043204

\section{INTRODUCTION}

Friction is one of the most common natural phenomena. Around 2 million years ago, in the Middle Paleolithic period, our ancestors used the phenomenon to make fire [1]. Nowadays, friction is responsible for a large fraction of global energy consumption [2]. At almost all macroscopic interfaces, the force of friction $\left(F_{\mathrm{f}}\right)$ is proportional to the normal force $\left(F_{\mathrm{n}}\right)$ [3-9]; the ratio of the two forces is constant and known as the coefficient of friction $\left(\mathrm{CoF}=F_{\mathrm{f}} / F_{\mathrm{n}}\right)$. Single contact experiments and simulations have demonstrated that the proportionality between frictional force and normal force can emerge because increased normal force results in stronger atomic scale interlocking and thus proportionally more frictional force [10-12]. Alternatively, if the atomic scale interlocking is dominated by adhesion rather than by externally applied force, the frictional force may scale with the

\footnotetext{
*Corresponding author: b.weber@arcnl.nl

Published by the American Physical Society under the terms of the Creative Commons Attribution 4.0 International license. Further distribution of this work must maintain attribution to the author(s) and the published article's title, journal citation, and DOI.
}

area of real contact $[10,12]$. At macroscopic, multi-asperity interfaces, the contacting materials form asperity contacts and touch at the atomic scale within the area of real contact [9,13]. Analytical theories such as the classical Greenwood and Williamson model [14] and the Persson contact theory [15] describe the process of contact formation. They attempt to explain why the area of real contact is proportional to the normal force, relating this to the surface topography, mechanical properties, and in some cases adhesion [16-18]. Alternatively, contact between rough surfaces can be understood through boundary element calculations and molecular dynamics simulations [16,18-20]. However, these theoretical approaches toward describing multi-asperity contact formation are built on assumptions - such as idealized elasticity and plasticity or frictionless contacts - that do not necessarily hold in reality [6]. The area of real contact is elusive and difficult to access experimentally because it is hidden from view [21,22] by the contacting materials and defined by the deformation of small-scale surface roughness variations. Therefore, it is challenging to compare multi-asperity contact theories to experiments at the appropriate length scales and even harder to assess the impact of the contact mechanics on friction [23]. As a result, a key question remained unanswered: to what extent does adhesive multi-asperity friction really depend on the area of real contact? 


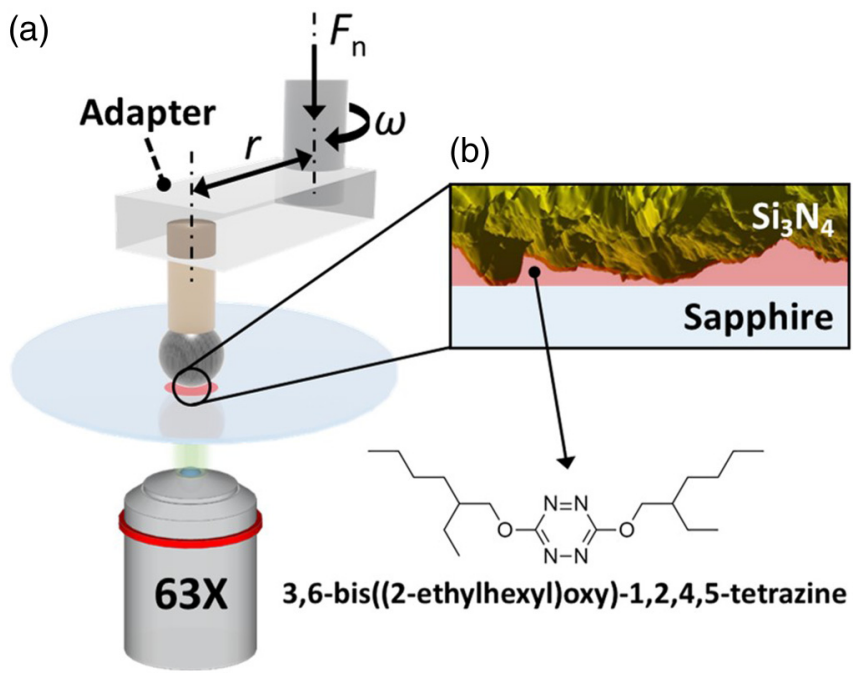

FIG. 1. Experimental setup. (a) A rheometer is mounted on top of an inverted confocal microscope. Via a rigid adapter, the rheometer controls the movement of a $\mathrm{Si}_{3} \mathrm{~N}_{4}$ sphere in contact with a sapphire flat. The sapphire flat has a root mean square roughness $\left(S_{\mathrm{q}}\right)$ of $0.14 \mathrm{~nm}$, measured by atomic force microscopy (AFM) over an area of $10 \times 10 \mu \mathrm{m}\left(95.4 \mathrm{~nm}^{2}\right.$ per pixel). By lowering and rotating the rheometer, a normal force $\left(F_{\mathrm{n}}\right)$ is applied at the $\mathrm{Si}_{3} \mathrm{~N}_{4}$-on-sapphire interface, and the torque $(\tau)$ is measured. In the contact visualization experiment, the contact is wetted with a droplet of fluorescent liquid, as shown in (b), where the inset illustrates the structure of the fluorescent liquid 3,6-bis[(2-ethylhexyl)oxy]-1,2,4,5-tetrazine. The fluorescent molecules are excited by $514 \mathrm{~nm}$ laser light, and the fluorescence intensity at the interface is measured by the microscope (63× magnification with 1.4 numerical aperture).

Here, we use a fluorescence microscopy-based contact visualization method to reveal the local nanometric gaps at a multi-asperity interface. Through a detailed comparison between visualization experiments and boundary element contact calculations, we show that the observed deformations of nanoscale surface roughness are well-described by idealized elastoplasticity. We show that a direct consequence of the contact mechanics is that the area of real contact will increase when the surface roughness is decreased and decrease when the roughness is increased. Counterintuitively, the surface roughness and area of real contact almost do not influence the dynamic frictional force: smooth and rough surfaces have approximately the same $\mathrm{CoF}$. However, when the interface roughness drops to values of just a few nanometers, we do find an increased $\mathrm{CoF}$ due to the large areas across which the interface is subject to capillary adhesion forces. These measurements give a comprehensive picture of how surface topography, elastoplasticity, and adhesion control the friction of multi-asperity contacts.

\section{RESULTS}

In our experiments, a customized rheometer pressed a 3.18mm-diameter $\mathrm{Si}_{3} \mathrm{~N}_{4}$ sphere against a sapphire flat plate at millinewton to Newton normal forces (Fig. 1). The $\mathrm{Si}_{3} \mathrm{~N}_{4}$ sphere was chosen because it has a similar hardness (23 GPa) to sapphire $(20 \mathrm{GPa})$, enabling elastic contact up to very high contact pressures and minimizing wear at the interface. To determine the area of real contact, we recorded fluorescence images of the interface using laser scanning confocal microscopy through the transparent sapphire substrate [24]. A droplet of low-viscosity fluorescent liquid 3,6-bis[(2ethylhexyl)oxy]-1,2,4,5-tetrazine [25] was placed in between the $\mathrm{Si}_{3} \mathrm{~N}_{4}$ sphere and sapphire flat before the normal force was applied. The large contact pressure associated with $\mathrm{Si}_{3} \mathrm{~N}_{4}$ on-sapphire contacts (GPa) in combination with the viscosity [26] of the fluorescent liquid (2000 Pas) ensured that viscous effects did not contribute to the transmission of the contact force at the experimental time scale $(>1 \mathrm{~s})$, as found previously [24]. If the local interfacial gap is large enough to host a liquid molecule ( $\sim 3 \mathrm{~nm}$ in size), an increased fluorescence intensity results. The relation between local interfacial gap and fluorescence intensity can be calibrated such that the local gap can be measured with nanometer accuracy (see Appendix A for more details) [27]. Combined with the submicrometer inplane imaging resolution, fluorescence microscopy reveals the multi-asperity contacts as no other technique can. We find that fluorescence images of the $\mathrm{Si}_{3} \mathrm{~N}_{4}$-on-sapphire contacts indeed display a clear peak in the intensity distribution at low but nonzero fluorescence intensities (Fig. S2 in the Supplemental Material [28]), corresponding to the area of real contact. Both detector noise and the convolution of contact and noncontact regions lead to the finite intensity measured at the area of real contact; pixels may contain a mixture of contact and no contact. To extract the area of real contact, we set an intensity threshold at a value that corresponds to an average gap of $6 \mathrm{~nm}$. Figure 2 shows that the area of real contact in the experiment increases with the normal force, as the asperities and bulk materials are being deformed. To compare these experimental observations with theoretical predictions of the contact formation process, we also performed contact calculations using the boundary element method (BEM) [19] (see Appendix B). Based on the sphere surface topography [measured using atomic force microscopy (AFM)] and the elastoplastic properties of $\mathrm{Si}_{3} \mathrm{~N}_{4}$ and sapphire (Table $\mathrm{SI}$ in the Supplemental Material [28]), the simulator solves the elastic equations and permits idealized plastic flow to relax the local contact pressure to values below the material hardness. Despite these idealizations of the contact problem, we find a compelling match between theory and experiment; not only does the predicted contact area correspond to what we observe experimentally, but individual contact patches revealed by fluorescence microscopy are reproduced by the calculations. This indicates that elastoplasticity is the key ingredient required to describe the $\mathrm{Si}_{3} \mathrm{~N}_{4}$-on-sapphire contact mechanics at the length scales that can be resolved by our inverted confocal fluorescence microscope.

A direct consequence of the elasticity of the asperities is that the area of real contact depends on the topography of the contacting surfaces; the smoother the surfaces become, the larger the area of real contact. To investigate the interplay between roughness and contact area deeper, we compared $\mathrm{Si}_{3} \mathrm{~N}_{4}$ spheres with different surface roughness as prepared by abrasion or polishing. We quantified the surface roughness either through the root mean square (RMS) height variation $\left(S_{\mathrm{q}}=99.3-4.7 \mathrm{~nm}\right)$ or through the RMS surface slope $\left(S_{\mathrm{k}}=\right.$ $0.45-0.06$ ) and the power spectral density (Fig. S3 in the 


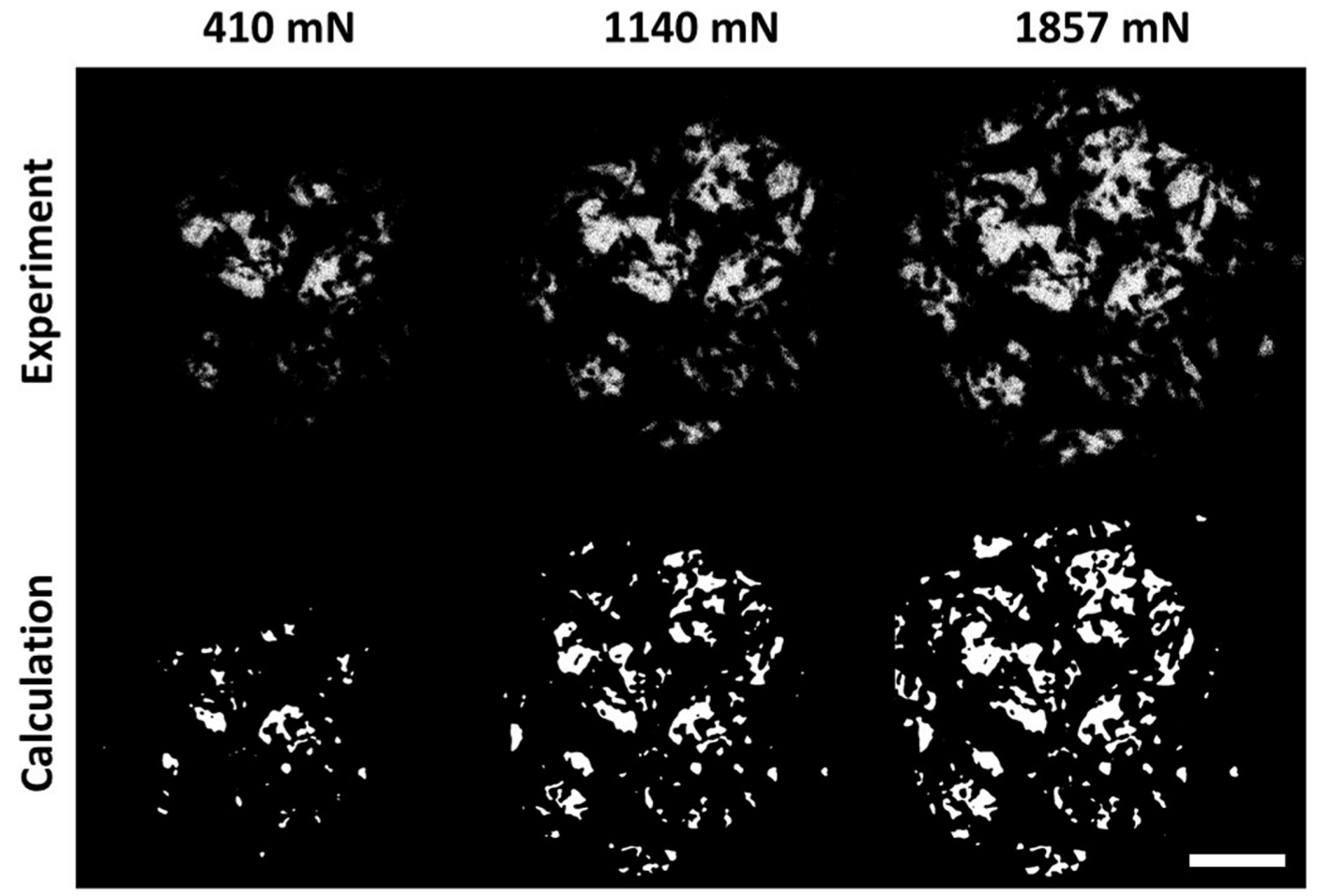

FIG. 2. $\mathrm{Si}_{3} \mathrm{~N}_{4}$-on-sapphire contact visualization and calculation. Upper row, area of real contact (white patches), defined as the pixels within which the average gap is smaller than $6 \mathrm{~nm}$, for three values of the normal force. Bottom row, the area of real contact (white patches) calculated by the Tribology Simulator based on the topography of the sphere used in the experiments as measured by atomic force microscopy (AFM; $60 \times 60 \mu \mathrm{m}, 2 \times 10^{3} \mathrm{~nm}^{2}$ per pixel). The results of the contact calculation were convolved with the microscopy point spread function: a Gaussian function with full width at half maximum of $600 \mathrm{~nm}$. Scale bar, $20 \mu \mathrm{m}$.

Supplemental Material [28]) [29], measured by AFM over an area of $10 \times 10 \mu \mathrm{m}$ with a pixel size of $95.4 \mathrm{~nm}^{2}$. Subsequently, we used the measured topographies to perform BEM contact calculations [such as shown in the inset of Fig. 3(a)] to quantify $A_{\text {real }}$ for each sphere roughness at various normal forces. As shown in Fig. 3(a), we find that there is a linear relation between the area of real contact and the normal force for each sphere. As expected, the area of real contact increases with decreasing surface roughness at the same normal force; the smoothest sphere makes contact over an area that is $\sim 4$ times larger than the area of real contact associated with the roughest sphere. To investigate if the larger area of real contact also leads to a larger frictional force, we carried out dry friction experiments with the $\mathrm{Si}_{3} \mathrm{~N}_{4}$ spheres with varying surface roughness (see Appendix $\mathrm{C}$ ). The rough-on-smooth contact geometry ensures that ploughing and interlocking friction are suppressed. We find that, despite the significant impact the roughness has on the contact mechanics [Fig. 3(a)], the variation in $\mathrm{CoF}$ is quite modest [Fig. 3(b)]. Reducing the area of real contact increases the interfacial normal stress and the interfacial shear stress such that the CoF varies much less than the contact pressure. This behavior contrasts with the classical description of friction by Bowden and Tabor [12] in which the friction force is proportional to the area of real contact. Proportionality between interfacial normal stress and interfacial shear stress has previously been observed in surface force apparatus experiments [10] and atomistic simulations $[11,30]$ and is in fact expected for stiff materials such as those used in the present experiments [12]. However, when averaged over the measured normal forces, the average CoF does show a small but systematic dependence on the surface roughness of the sphere [Fig. 3(b) inset].

To elucidate the observed relation between surface roughness and $\mathrm{CoF}$, we considered the contact calculations presented in Fig. 3 and analyzed these calculations in terms of the interfacial regions across which the gap between the two surfaces is finite but small enough for adhesive interactions to take place. Since the experimental interfaces were in ambient air, the surfaces were likely covered by water layers that can form capillary bridges around contact points [31]. The work of adhesion associated with capillarity $[32,33]$ can be as high as two times the water surface tension $\left(2 \gamma_{\text {water }}=145 \mathrm{~mJ} / \mathrm{m}^{2}\right)$, far above the work of adhesion associated with van der Waals interactions, which in turn decreases when the solid-on-solid interface is partially wetted by water [34]. The typical interfacial gap across which capillary bridges can be formed under thermodynamic equilibrium conditions is given by $W=$ $2 t+d_{\mathrm{c}}$. Here, $t$ is the equilibrium thickness of the water film condensed at a free silicon oxide $\left(\mathrm{SiO}_{x}\right)$ surface $(0.7 \mathrm{~nm}$ at $20 \%$ relative humidity ( $\mathrm{RH})$ and $1.1 \mathrm{~nm}$ at $50 \% \mathrm{RH}[35]$ ), and $d_{\mathrm{c}}$ is the critical distance between two surfaces at which capillary condensation occurs according to the Kelvin-Tolman equation [36] [1.5 nm at $20 \% \mathrm{RH}$ and $2.5 \mathrm{~nm}$ at $50 \% \mathrm{RH}$ (Table I)]. This yields interface gaps between $2.8 \mathrm{~nm}(20 \%$ $\mathrm{RH})$ and $4.8 \mathrm{~nm}(50 \% \mathrm{RH})$. A schematic illustration of the capillary bridges formed at the interface is shown in the inset of Fig. 4(a). There indeed is a dramatic increase in the area of the interface within which the surfaces are separated by a 

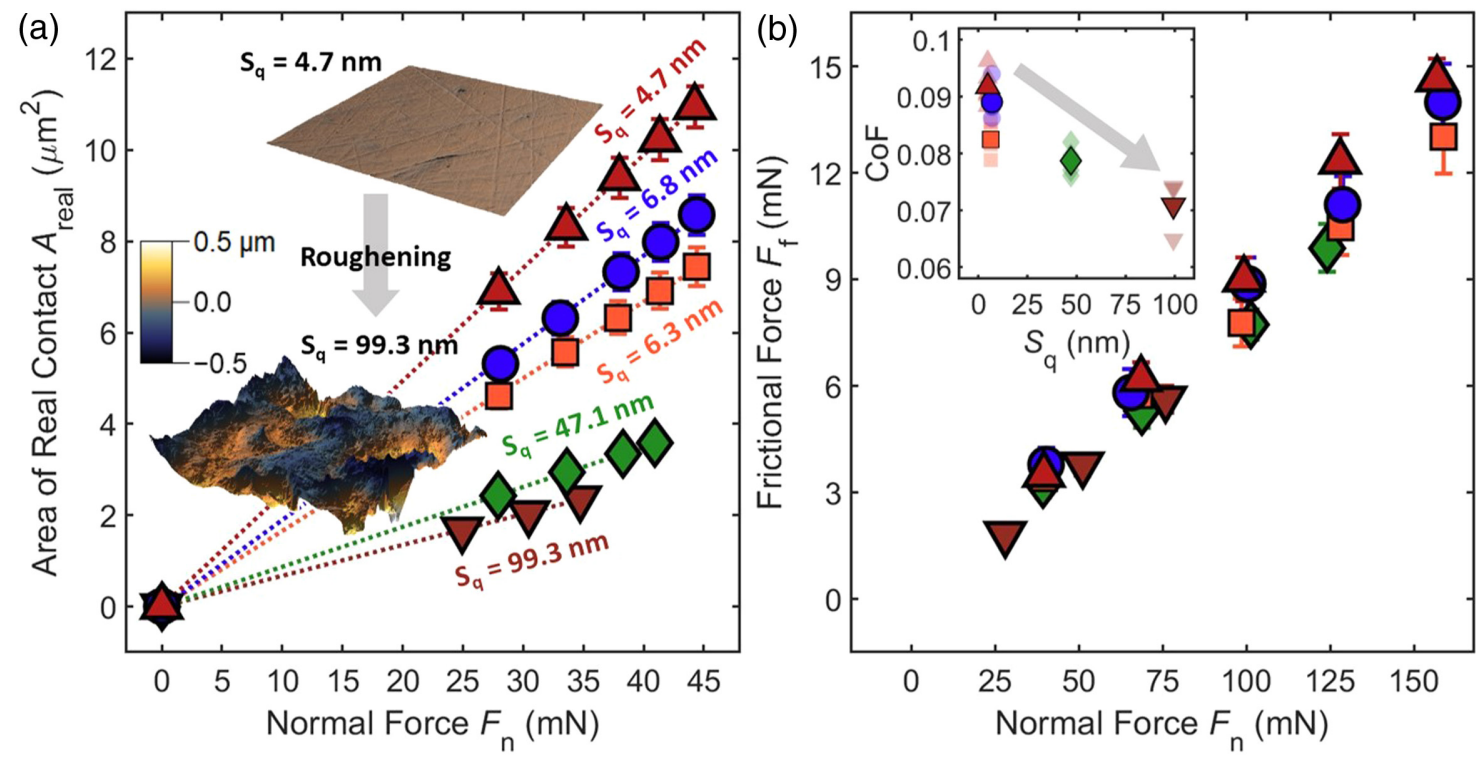

FIG. 3. Area of real contact and frictional force as a function of normal force and surface topography. (a) Calculated area of real contact $\left(A_{\text {real }}\right)$ as a function of normal force $\left(F_{\mathrm{n}}\right)$ for $\mathrm{Si}_{3} \mathrm{~N}_{4}$ spheres of varying roughness. The insets highlight $10 \times 10 \mu \mathrm{m}\left(95.4 \mathrm{~nm}{ }^{2}\right.$ per pixel) atomic force microscopy (AFM) topographies of the smoothest sphere $\left(S_{\mathrm{q}}=4.7 \mathrm{~nm}\right)$ and the roughest sphere $\left(S_{\mathrm{q}}=99.3 \mathrm{~nm}\right)$. (b) The frictional force $\left(F_{\mathrm{f}}\right)$ was measured at different normal forces $\left(F_{\mathrm{n}}\right)$ for the same spheres used in (a). The coefficient of friction $(\mathrm{CoF})$, averaged over the measured normal forces, is plotted in the inset. Contact calculations (a) were performed at normal forces up to $45 \mathrm{mN}$ to ensure that the nominal pressure in the calculations remained like that in the experiment (b). The error bars cover one standard deviation of the results.

finite gap that is $<4.8 \mathrm{~nm}$ [gray area Fig. 4 (b)] as the surface roughness decreases, suggesting that capillary adhesion may play a role in the observed dependence of the frictional force on the surface roughness.

Inspired by previous work [37], we now present a simplified model in which capillary adhesion occurs within gaps at the interface $<4.8 \mathrm{~nm}$, as illustrated by the gray areas in Fig. 4(b). In our model, we solely account for the capillary adhesion force and ignore other contributions to the adhesion such as the tension force and the van der Waals force. The capillary bridges at the interface are characterized by a positive radius of curvature $\left(r_{1}\right)$ in the in-plane direction and a negative radius of curvature $\left(r_{2}\right)$ in the direction normal to the hydrophilic interface. Because $\left|r_{1}\right| \gg\left|r_{2}\right|$ [see Fig. 4(b)], the overall radius of curvature of the capillary bridges $1 / R=$ $1 / r_{1}+1 / r_{2}$ is negative and dominated by $r_{2}$ such that a negative Laplace pressure difference between the water inside the bridges and the surrounding air leads to an adhesive force at the interface. The Laplace pressure $\left(P_{\text {Laplace }}\right)$ is given by $P_{\text {Laplace }}=\gamma_{\text {water }} /\left(r_{2}+\delta\right)$, with $\gamma_{\text {water }}$ the bulk water surface tension, $r_{2}=-d_{\mathrm{c}} / 2$, and $\delta$ the Tolman length $(\delta=0.2 \mathrm{~nm}$

TABLE I. Equilibrium thickness $(t)$ of the water condensate on a free $\mathrm{SiO}_{x}$ surface and critical distance for capillary condensation $\left(d_{\mathrm{c}}\right)$ of water as a function of relative humidity $(\mathrm{RH})$ as extracted from the literature [34,35].

\begin{tabular}{lcc}
\hline \hline RH $(\%)$ & $t(\mathrm{~nm})$ & $d_{\mathrm{c}}$ \\
\hline 20 & 0.65 & 1.49 \\
50 & 1.11 & 2.54 \\
\hline \hline
\end{tabular}

at $20-50 \%$ RH) [36]. Hence, the total adhesion force $\left(F_{\mathrm{ad}}\right)$ in our model is given by the product of the Laplace pressure difference across the water-air interface and the area on the sphere $\left(A_{\text {cap }}\right)$ that is wetted by the capillary water [Fig. 4(b)]; $F_{\text {ad }}=P_{\text {Laplace }} \times A_{\text {cap }}$. The capillary adhesion force associated with the modeled interface can therefore be calculatedwithout adjustable parameters-based on literature values for the water layer thickness on the free $\mathrm{SiO}_{x}$ surface, the critical distance for capillary condensation, the water surface tension, and the Tolman length. Through our model, we obtain capillary adhesion forces in the range of $3.9-14.5 \mathrm{mN}$ for the smoothest interface to $0.03-0.2 \mathrm{mN}$ for the roughest interface between $20 \% \mathrm{RH}$ and $50 \% \mathrm{RH}$.

The capillary adhesion-which is particularly strong for the smooth interface-contributes to the externally applied load $\left(F_{\text {ex }}\right)$ and is balanced by the repulsive force $\left(F_{\text {rep }}\right)$ generated at the solid-on-solid contacts: $F_{\text {rep }}=F_{\text {ex }}+F_{\text {ad }}$. As illustrated by the results in Fig. 3, the frictional force is proportional to the applied normal force. Therefore, the relative increase in frictional force resulting from capillary adhesion should scale as $F_{\text {ad }} / F_{\text {ex }}$. This ratio is close to zero for the roughest sphere: $F_{\mathrm{ad}} / F_{\mathrm{ex}}=6 \times 10^{-3}$. We thus can express the expected increase in $\mathrm{CoF}$ due to capillary adhesion $\left(\mathrm{CoF}_{\mathrm{ad}}\right)$ in terms of the $\mathrm{CoF}$ measured using the roughest sphere $\left(\mathrm{CoF}_{\text {roughest }}\right)$ :

$$
\mathrm{CoF}_{\mathrm{ad}}=\mathrm{CoF}_{\text {roughest }} \times\left(1+\frac{F_{\mathrm{ad}}}{F_{\mathrm{ex}}}\right) .
$$

Implicit in this calculation is the assumption that our thermodynamic equilibrium estimate of the capillary force holds during low-velocity rough-on-smooth sliding. Our simple adhesion model is thus used to predict the surface topography 

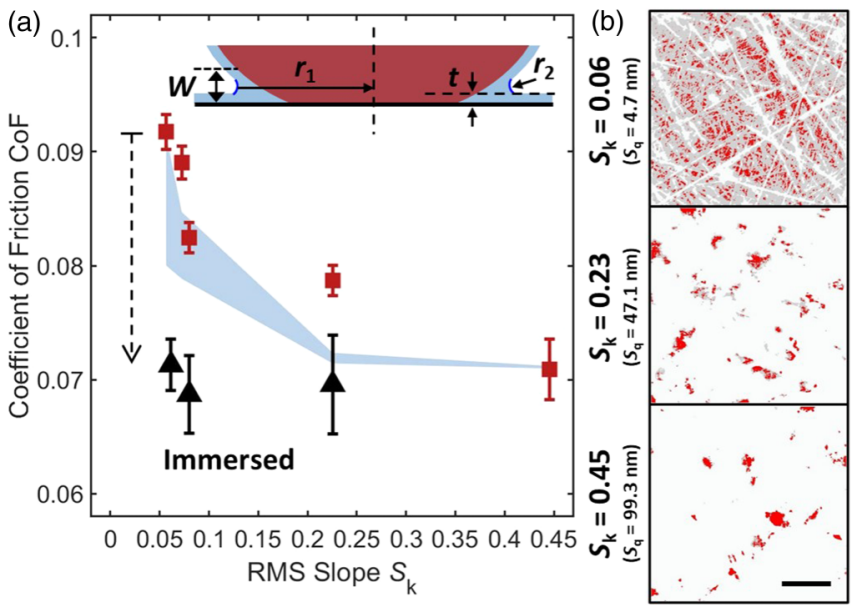

FIG. 4. Coefficient of friction $(\mathrm{CoF})$ as a function of surface slope and the effect of capillary adhesion. (a) $\mathrm{Si}_{3} \mathrm{~N}_{4}$-on-sapphire $\mathrm{CoF}$ (red square symbols) as measured using spheres with different topographies as a function of average root mean square (RMS) surface slope $\left(S_{\mathrm{k}}\right)$ of sphere surfaces as measured by atomic force microscopy (AFM). The gray shaded area shows the CoF values predicted by Eq. (1) for relative humidities ranging from 20 to $50 \%$. The black triangles indicate the $\mathrm{CoF}$ measured when the interface is immersed in water. The inset illustrates the formation of the capillary bridges at the multi-asperity interface. (b) Calculated contact areas at an externally applied normal force $\left(F_{\text {ex }}\right)$ of $35 \mathrm{mN}$ for three different $\mathrm{Si}_{3} \mathrm{~N}_{4}$ spheres with RMS slopes ranging from 0.06 (top) to 0.45 (bottom). The red and gray patches indicate solid-solid contact area and capillary-wetted area, respectively. The error bar covers the RMS variation between independent results. Scale bar, $2.5 \mu \mathrm{m}$.

dependence of the $\mathrm{CoF}$. The parameters that go into our model are not adjustable; the topography is measured by AFM, the BEM contact calculations are based on the elastoplastic properties of $\mathrm{Si}_{3} \mathrm{~N}_{4}$ and sapphire, and the capillary adhesion range and Laplace pressure are estimated based on the Kelvin-Tolman equation and based on previous measurements [35]. We evaluate the model for RHs in the typical range for lab experiments $(\mathrm{RH}=20-50 \%)$ and plot the results in Fig. 4(a). The predicted $\mathrm{CoF}\left(\mathrm{CoF}_{\mathrm{ad}}\right)$-without adjustable parameters - quantitatively captures the dependence of the $\mathrm{CoF}$ on surface roughness: smoother interfaces display higher frictional force due to capillary adhesion. As capillary adhesion can only take place in the presence of solid, liquid, and gas phases, we further challenge our model by immersing the contacts in liquid water. This removes the air from the contact zone, preventing capillary bridges from forming. Indeed, this results in a $\mathrm{CoF}$ that is no longer dependent on the surface roughness [Fig. 4(a)], confirming our model. We want to emphasize that this provides direct evidence that all dependence of the $\mathrm{CoF}$ on the area of real contact in ambient conditions can be attributed to capillary effects and that, therefore, the experiments and model provide indirect evidence that the shear stress depends linearly on the normal stress (Fig. S5 in the Supplemental Material [28]).

While our results demonstrate how surface topography affects dynamic friction through capillary adhesion, the impact of surface topography on static friction remains an

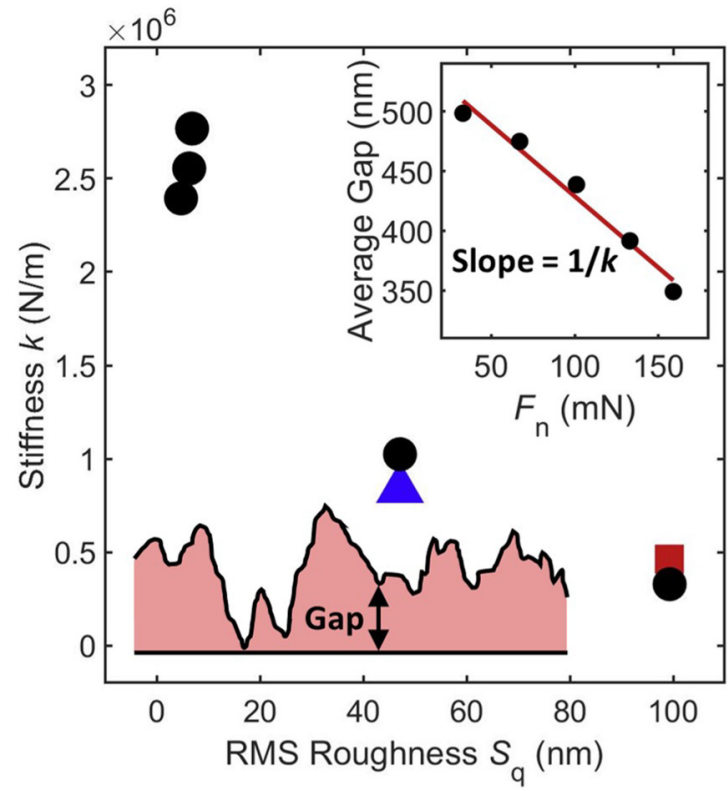

FIG. 5. Normal contact stiffness as a function of surface roughness. Black circular symbols indicate boundary element method (BEM) contact calculations based on the sphere topographies. The blue triangle and red square symbols indicate measurements using the contact visualization method. The measured average gap at the multi-asperity interface (an example is shown in the bottom left inset) is shown in the upper right inset as a function of normal force $\left(F_{\mathrm{n}}\right)$. For the smoothest interfaces, the average gaps become comparable with the size of the fluorescent molecules $(\sim 3 \mathrm{~nm})$, and the visualization experiments can no longer reliably measure interface stiffness.

important open question. The transition from static to dynamic friction [38-40] is of key importance to, for instance, the initiation of earthquakes or the precision and accuracy of positioning systems and microelectromechanical system or nanoelectromechanical system actuators. A loaded interface subjected to an increasing tangential force will initially deform elastically before the interfacial junctions yield. However, the asperity-scale deformations associated with this presliding process are typically difficult to assess experimentally. Nonetheless, the tangential interface stiffness that determines the initial elastic response of an interface to an increasing tangential force is predicted to be proportional to the normal stiffness of the interface [41]. The combination of experiments and contact calculations employed in this paper enables a direct measurement of this normal interfacial stiffness as a function of surface topography. In the contact calculations discussed above, we extracted the average interfacial gap (Gap) as a function of the normal force and derived the normal interfacial contact stiffness $(k)$ using Hooke's law, $k=F_{\mathrm{n}} /$ Gap. In these calculations, we only consider the roughness of the $\mathrm{Si}_{3} \mathrm{~N}_{4}$ sphere, as the RMS roughness of the sapphire plate is two orders of magnitude smaller. Rougher interfaces-higher $S_{\mathrm{q}}$ or $S_{\mathrm{k}}$ of $\mathrm{Si}_{3} \mathrm{~N}_{4}$ sphere-on-sapphire flathave a lower interfacial stiffness than smooth interfaces. The results show that the normal contact stiffness strongly depends on the RMS roughness of the sphere surface (Fig. 5). Since the contact pressure for most spheres is below the hardness of the 
contacting materials (see also Fig. S6 in the Supplemental Material [28]), the contact stiffness is determined by the elasticity of the long wavelength roughness of the surface. The fluorescence imaging of the experimental interface provides an additional opportunity to measure the average interfacial gap as a function of normal force, through the average interfacial fluorescence intensity (Fig. 5; see also Appendix D). The contact stiffness measured in the visualization experiments agrees with the contact calculations and far beyond the stiffness of most mechanical measurement devices.

\section{DISCUSSION}

In conclusion, we demonstrated that the $\mathrm{Si}_{3} \mathrm{~N}_{4}$-on-sapphire frictional force is not proportional to the area of real contact; the $\mathrm{CoF}$ reflects a proportionality between normal stress and shear stress. By controlling the surface topography, we varied the area of real contact between a $\mathrm{Si}_{3} \mathrm{~N}_{4}$ sphere and flat sapphire surface by a factor of four and found a much weaker but systematic variation in $\mathrm{CoF}$ of $\sim 20 \%$. This $20 \%$ variation of the CoF with surface roughness was found to be caused by capillary adhesion; the smoother the interface, the larger the area across which capillary adhesion can contribute to the load experienced by the contacting asperities. Our simple adhesion model, based on the Laplace and Kelvin-Tolman equations and without adjustable parameters, successfully predicts the adhesion-induced increase in the $\mathrm{CoF}$ with decreasing surface roughness for RHs in the range of 20-50\%. This large range of humidity reflects that the model can capture the experimental trend regardless of the precise thickness of the preabsorbed water film, which may vary for different hydrophilic surfaces. In the calculations, the contact angle at the three-phase contact line is ignored; considering a small contact angle for the hydrophilic (oxidized) $\mathrm{Si}_{3} \mathrm{~N}_{4}$ and sapphire surfaces would result in a small decrease in the range of the capillary adhesion but would not affect the overall trend of increasing $\mathrm{CoF}$ for decreasing surface roughness. The solid-on-solid friction measured in the experiments may be affected by boundary layers of water and/or airborne hydrocarbons from the environment. Since the only material-specific parameters included in our model are the bulk elastoplastic properties, the model can in principle be applied to any stiff hydrophilic interface. Interfaces between materials with lower stiffness have been shown to display a more complex interplay between adhesion and friction [37,42]. While our microscopy and AFM-based approach does not enable the measurement of surface roughness at length scales smaller than that of the AFM tip [43], we conclude that the resolution is sufficient to capture the mechanism by which topography controls friction $[44,45]$ : capillary adhesion. In addition to showing that dynamic friction at such interfaces is largely independent on surface roughness, we also show that the elastic deformations that play an important role in controlling the initial response of an interface to external loading are highly roughness dependent; the interfacial stiffness was shown to change by almost an order of magnitude as the RMS slope varied from 0.06 to 0.45 (corresponding to RMS roughness range of 5-100 nm). These results provide insight into how friction behavior evolves as surface roughness is worn off, which is paramount in precision positioning applications.

\section{ACKNOWLEDGMENTS}

The work in this paper was carried out at ARCNL. This paper is part of the project "Friction on demand: to slide or not to slide" with Project No. VI.Veni.192.177, which is (partly) financed by the Dutch Research Council. We thank Laurent Galmiche for his help in preparing the fluorescent liquid and Cyrian Leriche for his help in preparing the spheres.

\section{APPENDIX A: CONTACT VISUALIZATION}

In the contact visualization experiments, a rheometer (DSR 301, Anton Paar) was placed on top of an inverted laser scanning confocal microscope (Axiovert 200M, Zeiss) with an LSM 5 PASCAL (Zeiss) laser scanning module [Fig. 1(a)]. A $3.18 \mathrm{~mm} \mathrm{Si}_{3} \mathrm{~N}_{4}$ ball was fixed to the rheometer and brought into contact with the sapphire flat $(22 \mathrm{~mm}$ diameter), which was mounted on the microscopy sample stage. A droplet of fluorescent liquid 3,6-bis[(2-ethylhexyl)oxy]-1,2,4,5-tetrazine was placed between the $\mathrm{Si}_{3} \mathrm{~N}_{4}$ sphere and sapphire flat. A 514 $\mathrm{nm}$ laser light was used to excite the fluorescent molecules. The fluorescence images were recorded using a $63 \times 1.4$ numerical aperture objective (Plan-Apochromat, Zeiss) at a pixel size of $\sim 3400 \mathrm{~nm}^{2}$. At the interface, the fluorescent molecules were excited by the laser light, and the local fluorescence intensity scales linearly with the local gap [27]. Figure S1 in the Supplemental Material [28] shows the fluorescence intensity measured across two orthogonal profiles that run through the center of the sphere-on-flat contact. To conduct these measurements, the $\mathrm{Si}_{3} \mathrm{~N}_{4}$ sphere was placed on the sapphire flat with no externally applied load — only gravitational force. Therefore, the deformation of the bulk sphere was negligible. The predicted sphere-on-flat gap, assuming there is no roughness and no contact deformation, is shown by the black solid and dotted curves in Fig. S1(a) in the Supplemental Material [28]. We subtracted the finite background fluorescence intensity. We find a linear relation between the intensity and the gap $<500 \mathrm{~nm}$ in both orthogonal in-plane directions (Fig. S1(b) in the Supplemental Material [28]). The intensity-gap proportionality constant can be found by averaging the slope of the linear fitting lines on two intensity directions $\sim 4.7 \mathrm{I} / \mathrm{nm}$.

\section{APPENDIX B: BEM CONTACT CALCULATION}

Contact calculations are carried out by the Tribology Simulator [46]. The half-space contact calculations were based on the BEM to estimate the elastoplastic deformation (strain hardening is neglected) at the $\mathrm{Si}_{3} \mathrm{~N}_{4}$ sphere-on-sapphire flat interface, where the input mechanical properties in the calculation are listed in Table SI in the Supplemental Material [28]. The best match between experiment and calculation was obtained when the calculated contact image was convolved with the microscopy point spread function, and subsequently, a gap threshold of $9 \mathrm{~nm}$ was set (Fig. 2). This is $3 \mathrm{~nm}$ larger than the gap used to obtain the experimental value of the contact area; the difference is of the order of the molecule size. We hypothesize that the difference may therefore be related to the inability of the fluorescent liquid molecules to fill the smallest gaps or the quenching of the fluorescence of the boundary layer molecules that interface to the solid sur- 
faces. Nonetheless, we want to emphasize that the presented comparison between contact calculation and experiment provides an unprecedented level of detail.

\section{APPENDIX C: FRICTION EXPERIMENTS}

The $\mathrm{Si}_{3} \mathrm{~N}_{4}$ sphere-on-sapphire flat friction experiments were performed using a customized rheometer setup (DSR 301, Anton Paar) placed in an ambient environment at $\sim 40 \%$ $\mathrm{RH}$ [Fig. 1(a)]. The 3.18-mm-diameter $\mathrm{Si}_{3} \mathrm{~N}_{4}$ sphere was slid against the sapphire flat at a constant angular velocity $\left(\omega=8.3^{*} 10^{-5} \mathrm{rad} / \mathrm{s}\right)$ that can be converted into a constant sliding speed by multiplication with the fixed rotation radius $(r=12.98 \mathrm{~mm}) ; V=\omega r=1 \mu \mathrm{m} / \mathrm{s}$. The torque $(\tau)$ experienced while sliding was measured and converted to the frictional force $\left(F_{\mathrm{f}}=\tau / r\right) . \mathrm{Si}_{3} \mathrm{~N}_{4}$ spheres and sapphire flats were cleaned by sonicating with isopropanol solution and kept in an ambient environment before friction experiments. The friction experiments were performed at different normal forces indicated in Fig. 3(b) with different $\mathrm{Si}_{3} \mathrm{~N}_{4}$ sphere topographies. The maximum normal force applied in the experiment was $160 \mathrm{mN}$, which results in a Hertzian contact pressure of $\sim 0.6 \mathrm{GPa}$. The Hertzian contact pressure is much lower than the hardness (Table SI in the Supplemental Material [28]) of $\mathrm{Si}_{3} \mathrm{~N}_{4}$ and sapphire so that the chance of plastic deformation and ploughing was minimized. In addition, in Fig. S4 in the Supplemental Material [28], we demonstrate the AFM topography of a $\mathrm{Si}_{3} \mathrm{~N}_{4}$ sphere before and after the contact with a sapphire flat at $2 \mathrm{~N}$ normal force. At these elevated normal forces, we do observe some plasticity. To correct for possible tilting of the rheometer with respect to the substrate, we slid the $\mathrm{Si}_{3} \mathrm{~N}_{4}$ sphere on the sapphire flat both in the positive and negative directions and reported the average frictional force measured in both directions under the same applied normal force. For each normal force, the sliding strokes were performed at a previously untouched piece of the sapphire flat to prevent potential interaction of the contact surfaces and wear debris [47]. The frictional force at each normal force was measured at least three times (six times in both directions) by following the applied normal force sequence: increase, decrease, then increase. No hysteresis was observed, confirming that wear was minimized.

\section{APPENDIX D: CONTACT STIFFNESS MEASUREMENT AND CALCULATION}

The contact stiffness measurements were conducted using the same experimental setup as described above. The fluorescence intensity was averaged within the Hertzian contact area $\left(A_{\mathrm{Hertz}}\right)$ between the $\mathrm{Si}_{3} \mathrm{~N}_{4}$ sphere and sapphire flat at different normal forces:

$$
\mathrm{A}_{\mathrm{Hertz}}=\pi\left(\frac{3 F_{\mathrm{n}} R}{4 E_{\mathrm{eff}}}\right)^{2 / 3},
$$

where $R(=1.59 \mathrm{~mm})$ is the radius of $\mathrm{Si}_{3} \mathrm{~N}_{4}$ sphere and $E_{\text {eff }}=$ $\left(1-v_{1} / E_{1}+1-v_{2} / E_{2}\right)^{-1}$ is the effective modulus of $\mathrm{Si}_{3} \mathrm{~N}_{4}$ (labeled as 1) and sapphire (labeled as 2). The mechanical properties of $\mathrm{Si}_{3} \mathrm{~N}_{4}$ and sapphire are listed in Table SI in the Supplemental Material [28]. The average fluorescence intensity (a.u.) was converted to a gap value (nm) by using the intensity-gap calibration as discussed above. Contact stiffness calculations were done using the BEM. The $\mathrm{Si}_{3} \mathrm{~N}_{4}$ sphere topography was measured by laser scanning confocal microscopy (VK-X1000, Keyence) with a pixel size of $\sim 76 \times 10^{3} \mathrm{~nm}^{2}$. The average gap values were based on the interfacial gap map output by the BEM calculation at normal forces between 30 and $160 \mathrm{mN}$ with $S_{\mathrm{k}}=0.23$ sphere (blue triangle symbol) and $30-120 \mathrm{mN}$ with $S_{\mathrm{k}}=0.45$ sphere (red square symbol) in Fig. 5. In Fig. S6 in the Supplemental Material [28], we report the contact stiffness calculated for the roughest sphere in the absence of plasticity. As the sharp asperity peaks are not flattened plastically under these conditions, the interface is much more compliant.

\section{APPENDIX E: AFM SURFACE TOPOGRAPHY CHARACTERIZATION}

The surface topography characterization was performed using AFM (Dimension Icon, Bruker) in tapping mode. Power spectral density analysis of the topographies are presented in Fig. S3 in the Supplemental Material [28]. A silicon AFM tip with an apex of $\sim 8 \mathrm{~nm}$ radius (RTESPA-300, Bruker) was used and replaced for each topography measurement.
[1] W. Roebroeks and P. Villa, On the earliest evidence for habitual use of fire in Europe, Proc. Natl. Acad. Sci. U.S.A. 108, 5209 (2011).

[2] K. Holmberg and A. Erdemir, Influence of tribology on global energy consumption, costs and emissions, Friction 5, 263 (2017).

[3] I. M. Hutchings, Leonardo da Vinci's studies of friction, Wear 360-361, 51 (2016).

[4] E. Popova and V. L. Popov, The research works of Coulomb and Amontons and generalized laws of friction, Friction 3, 183 (2015).

[5] C. M. Mate and R. W. Carpick, Tribology on the Small Scale: A Modern Textbook on Friction, Lubrication and Wear, 2nd ed. (Oxford University Press, Oxford, 2019).
[6] B. Weber, T. Suhina, T. Junge, L. Pastewka, A. M. Brouwer, and D. Bonn, Molecular probes reveal deviations from Amontons' law in multi-asperity frictional contacts, Nat. Commun. 9, 888 (2018).

[7] R. W. Carpick, D. F. Ogletree, and M. Salmeron, A general equation for fitting contact area and friction vs load measurements, J. Colloid Interface Sci. 211, 395 (1999).

[8] J. Gao, W. D. Luedtke, D. Gourdon, M. Ruths, J. N. Israelachvili, and U. Landman, Frictional forces and Amontons' law: From the molecular to the macroscopic scale, J. Phys. Chem. B 108, 3410 (2004).

[9] X. He, Z. Liu, L. B. Ripley, V. L. Swensen, I. J. GriffinWiesner, B. R. Gulner, G. R. McAndrews, R. J. Wieser, B. P. Borovsky, Q. J. Wang, and S. H. Kim, Empirical relationship 
between interfacial shear stress and contact pressure in microand macro-scale friction, Tribol. Int. 155, 106780 (2021).

[10] A. Berman, C. Drummond, and J. Israelachvili, Amontons' law at the molecular level, Tribol. Lett. 4, 95 (1998).

[11] G. He and M. H. Müser, Adsorbed layers and the origin of static friction, Science 284, 1650 (1999).

[12] F. P. Bowden and D. Tabor, The Friction and Lubrication of Solids (Oxford University Press, Oxford, 2001), Vol. 1.

[13] M. Enachescu, R. J. A. Van Den Oetelaar, R. W. Carpick, D. F. Ogletree, C. F. J. Flipse, and M. Salmeron, Observation of proportionality between friction and contact area at the nanometer scale, Tribol. Lett. 7, 73 (1999).

[14] J. A. Greenwood and J. B. P. Williamson, Contact of nominally flat surfaces, Proc. R. Soc. London A 295, 300 (1966).

[15] B. N. J. Persson, Theory of rubber friction and contact mechanics, J. Chem. Phys. 115, 3840 (2001).

[16] L. Pastewka and M. O. Robbins, Contact between rough surfaces and a criterion for macroscopic adhesion, Proc. Natl. Acad. Sci. U.S.A. 111, 3298 (2014).

[17] B. N. J. Persson, Contact mechanics for randomly rough surfaces, Surf. Sci. Rep. 61, 201 (2006).

[18] L. Pastewka and M. O. Robbins, Contact area of rough spheres: Large scale simulations and simple scaling laws, Appl. Phys. Lett. 108, 221601 (2016).

[19] M. H. Müser, W. B. Dapp, R. Bugnicourt, P. Sainsot, N. Lesaffre, T. A. Lubrecht, B. N. J. Persson, K. Harris, A. Bennett, K. Schulze, S. Rohde, P. Ifju, W. G. Sawyer, T. Angelini, H. Ashtari Esfahani, M. Kadkhodaei, S. Akbarzadeh, J.-J. Wu, G. Vorlaufer, A. Vernes et al., Meeting the contact-mechanics challenge, Tribol. Lett. 65, 118 (2017).

[20] B. Luan and M. O. Robbins, The breakdown of continuum models for mechanical contacts, Nature (London) 435, 929 (2005).

[21] R. Sahli, G. Pallares, C. Ducottet, I. E. Ben Ali, S. Al Akhrass, M. Guibert, and J. Scheibert, Evolution of real contact area under shear and the value of static friction of soft materials, Proc. Natl. Acad. Sci. U.S.A. 115, 471 (2018).

[22] A. J. McGhee, A. A. Pitenis, A. I. Bennett, K. L. Harris, K. D. Schulze, J. M. Urueña, P. G. Ifju, T. E. Angelini, M. H. Müser, and W. G. Sawyer, Contact and deformation of randomly rough surfaces with varying root-mean-square gradient, Tribol. Lett. 65, 157 (2017).

[23] R. W. Carpick, The contact sport of rough surfaces, Science 359, 38 (2018).

[24] D. Petrova, B. Weber, C. Allain, P. Audebert, C. H. Venner, A. M. Brouwer, and D. Bonn, Fluorescence microscopy visualization of the roughness-induced transition between lubrication regimes, Sci. Adv. 5, eaaw4761 (2019).

[25] C. Allain, J. Piard, A. Brosseau, M. Han, J. Paquier, T. Marchandier, M. Lequeux, C. Boissière, and P. Audebert, Fluorescent and electroactive low-viscosity tetrazine-based organic liquids, ACS Appl. Mater. Interfaces. 8, 19843 (2016).

[26] M. P. Dominguez, B. Demirkurt, M. Grzelka, D. Bonn, L. Galmiche, P. Audebert, and A. M. Brouwer, Fluorescent liquid tetrazines, Molecules 26, 6047 (2021).

[27] D. Petrova, B. Weber, C. Allain, P. Audebert, D. Bonn, and A. M. Brouwer, Fast 3D microscopy imaging of contacts between surfaces using a fluorescent liquid, ACS Appl. Mater. Interfaces. 10, 40973 (2018).
[28] See Supplemental Material at http://link.aps.org/supplemental/ 10.1103/PhysRevResearch.3.043204 for more details of the experimental methods and results, surface characterization, and contact calculation.

[29] S. R. Khanal, A. Gujrati, S. B. Vishnubhotla, P. Nowakowski, C. S. Bonifacio, L. Pastewka, and T. D. B. Jacobs, Characterization of small-scale surface topography using transmission electron microscopy, Surf. Topogr. Metrol. Prop. 6, 045004 (2018).

[30] H. Sakuma, K. Kawai, I. Katayama, and S. Suehara, What is the origin of macroscopic friction? Sci. Adv. 4, eaav2268 (2018).

[31] M. D. Butler and D. Vella, Liquid bridge splitting enhances normal capillary adhesion and resistance to shear on rough surfaces, J. Colloid Interface Sci. 607, 514 (2022).

[32] P. J. van Zwol, G. Palasantzas, and J. T. M. De Hosson, Influence of random roughness on the adhesion between metal surfaces due to capillary condensation, Appl. Phys. Lett. 91, 101905 (2007).

[33] E. Soylemez and M. P. de Boer, Capillary-induced crack healing between surfaces of nanoscale roughness, Langmuir 30, 11625 (2014).

[34] M. Bartošík, L. Kormoš, L. Flajšman, R. Kalousek, J. Mach, Z. Lišková, D. Nezval, V. Švarc, T. Šamořil, and T. Šikola, Nanometer-sized water bridge and pull-off force in AFM at different relative humidities: Reproducibility measurement and model based on surface tension change, J. Phys. Chem. B 121, 610 (2017).

[35] D. B. Asay and S. H. Kim, Evolution of the adsorbed water layer structure on silicon oxide at room temperature, J. Phys. Chem. B 109, 16760 (2005).

[36] S. Kim, D. Kim, J. Kim, S. An, and W. Jhe, Direct Evidence for Curvature-Dependent Surface Tension in Capillary Condensation: Kelvin Equation at Molecular Scale, Phys. Rev. X 8, 041046 (2018).

[37] B. N. J. Persson, Capillary adhesion between elastic solids with randomly rough surfaces, J. Phys. Condens. Matter. 20, 315007 (2008).

[38] M. Bazrafshan, M. B. de Rooij, E. G. de Vries, and D. J. Schipper, Evaluation of pre-sliding behavior at a rough interface: Modeling and experiment, J. Appl. Mech. 87, 041006 (2020).

[39] B. Weber, T. Suhina, A. M. Brouwer, and D. Bonn, Frictional weakening of slip interfaces, Sci. Adv. 5, eaav7603 (2019).

[40] E. Bayart, I. Svetlizky, and J. Fineberg, Fracture mechanics determine the lengths of interface ruptures that mediate frictional motion, Nat. Phys. 12, 166 (2016).

[41] C. Campañá, B. N. J. Persson, and M. H. Müser, Transverse and normal interfacial stiffness of solids with randomly rough surfaces, J. Phys. Condens. Matter. 23, 085001 (2011).

[42] O. Lahayne, B. Pichler, R. Reihsner, J. Eberhardsteiner, J. Suh, D. Kim, S. Nam, H. Paek, B. Lorenz, and B. N. J. Persson, Rubber friction on ice: Experiments and modeling, Tribol. Lett. 62, 17 (2016)

[43] A. Gujrati, S. R. Khanal, L. Pastewka, and T. D. B. Jacobs, Combining TEM, AFM, and profilometry for quantitative topography characterization across all scales, ACS Appl. Mater. Interfaces. 10, 29169 (2018).

[44] L. A. Thimons, A. Gujrati, A. Sanner, L. Pastewka, and T. D. B. Jacobs, Hard-material adhesion: Which scales of roughness matter? Exp. Mech. 61, 1109 (2021). 
[45] M. Ciavarella and A. Papangelo, Discussion of "Measuring and Understanding Contact Area at the Nanoscale: A Review" (Jacobs, T. D. B., and Ashlie Martini, A., 2017, ASME Appl. Mech. Rev., 69(6), p. 060802), Appl. Mech. Rev. 69, 065502 (2017).
[46] Publicly available at www.tribology.org.

[47] F.-C. Hsia, F. M. Elam, D. Bonn, B. Weber, and S. E. Franklin, Wear particle dynamics drive the difference between repeated and non-repeated reciprocated sliding, Tribol. Int. 142, 105983 (2020). 